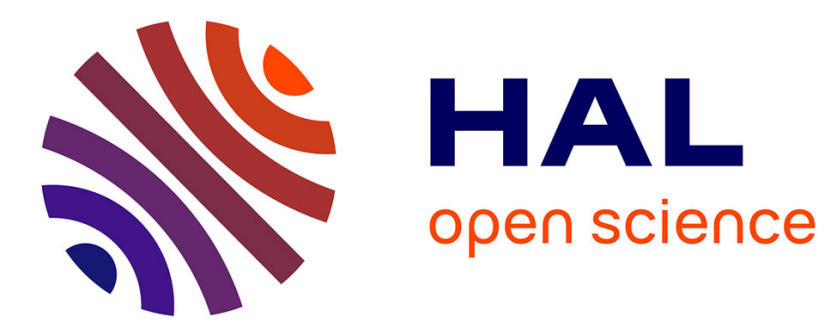

\title{
Development of magnetohydrodynamic modes during sawteeth in tokamak plasmas
}

\author{
Marie-Christine Firpo, Wahb Ettoumi, Ricardo Farengo, Hugo Ferrari, Pablo \\ Luis Garcia-Martinez, Agustin Lifschitz
}

\section{- To cite this version:}

Marie-Christine Firpo, Wahb Ettoumi, Ricardo Farengo, Hugo Ferrari, Pablo Luis Garcia-Martinez, et al.. Development of magnetohydrodynamic modes during sawteeth in tokamak plasmas. Physics of Plasmas, 2013, 20 (7), pp.072305. 10.1063/1.4816025 . hal-00846433

\section{HAL Id: hal-00846433 \\ https://hal.science/hal-00846433}

Submitted on 19 Jul 2013

HAL is a multi-disciplinary open access archive for the deposit and dissemination of scientific research documents, whether they are published or not. The documents may come from teaching and research institutions in France or abroad, or from public or private research centers.
L'archive ouverte pluridisciplinaire HAL, est destinée au dépôt et à la diffusion de documents scientifiques de niveau recherche, publiés ou non, émanant des établissements d'enseignement et de recherche français ou étrangers, des laboratoires publics ou privés. 


\title{
Development of magnetohydrodynamic modes during sawteeth in tokamak plasmas
}

\author{
M.-C. Firpo, ${ }^{1}$ W. Ettoumi, ${ }^{1}$ R. Farengo, ${ }^{2}$ H. E. Ferrari, ${ }^{2,3}$ P. L. García-Martínez, ${ }^{3}$ and A. F. Lifschitz ${ }^{4}$ \\ ${ }^{1)}$ Laboratoire de Physique des Plasmas, CNRS - Ecole Polytechnique, 91128 Palaiseau cedex, \\ France \\ ${ }^{2)}$ Centro Atómico Bariloche (CNEA) and Instituto Balseiro (UNC-CNEA), San Carlos de Bariloche, RN 8400, \\ Argentina \\ 3) Consejo Nacional de Investigaciones Científicas y Técnicas (CONICET), Bariloche, \\ Argentina \\ 4) Laboratoire d'Optique Appliquée, ENSTA, CNRS, Ecole Polytechnique, 91761 Palaiseau, \\ France
}

( Dated: 19 July 2013)

\begin{abstract}
A dynamical analysis applied to a reduced resistive magnetohydrodynamics model is shown to explain the chronology of the nonlinear destabilization of modes observed in tokamak sawteeth. A special emphasis is put on the nonlinear self-consistent perturbation of the axisymmetric $m=n=0$ mode that manifests through the $q$-profile evolution. For the very low fusion-relevant resistivity values, the $q$-profile is shown to remain almost unchanged on the early nonlinear timescale within the central tokamak region, which supports a partial reconnection scenario. Within the resistive region, indications for a local flattening or even a local reversed-shear of the $q$-profile are given. The impact of this ingredient in the occurrence of the sawtooth crash is discussed.
\end{abstract}

PACS numbers: 52.30.Cv,52.35.Py,52.35.Mw,52.55.Tn

\section{MOTIVATIONS}

One still largely open problem in tokamak plasmas is the generic "sawtooth" phenomenon. Sawteeth manifest e.g. with respect to the plasma temperature indicator in the following fashion: the core plasma temperature suddenly crashes when attaining some limit value before progressively increasing further up to the next sawtooth crash on a periodic basis, so that the resulting time trace of the central temperature resembles the edge of a saw. Despite an increasing phenomenological and theoretical knowledge, acquired since their first observation in the early $1970 \mathrm{~s}^{1}$, as well as some very recent experimental successfully tested procedure to control their pace $^{2}$, aimed at containing their deleterious effects on the plasma, it is widely admitted by the magnetic fusion community that the phenomenon of sawteeth is not fully understood yet.

This issue is of special concern in the perspective of ITER. Actually, whereas small sawteeth may even be beneficial in preventing the accumulation of impurities and helium ash in the plasma center, large sawteeth with mixing radii of $50 \%$ or more of the plasma minor radius and temperature drops of one or more $\mathrm{keV}$ represent a serious threat to ITER operation, since these large sawtooth events may couple to neoclassical tearing modes and to edge-localized modes, resulting in a serious loss of plasma energy and confinement degradation ${ }^{3}$. Moreover, from a fusion perspective, the central temperature in ITER should be as large as possible. The best performance is just before the plasma crashes. Understanding the nature of sawteeth should help in finding a way to delay the time between crashes and therefore sustain the hot temperature and get more fusion from ITER. Im- proving solely the modeling of sawteeth would be also useful for studying many effects occurring in the sawteeth regime such as the behavior of alpha particles ${ }^{4,5}$. In this respect, relying on a purely numerical approach may not be the most relevant approach or, at least, should be taken with care. Indeed the numerical modeling of sawteeth is well known to be a considerably difficult task. In particular, MHD based codes, even the most sophisticated ones, repeatedly predict a complete reconnection at each sawtooth cycle (in the absence of ad-hock tricks) whereas experiments have almost always reported an incomplete reconnection ${ }^{6}$.

It is widely admitted that the occurrence of sawteeth in tokamak plasmas is related to the existence of some $q=1$ internal magnetic surface and to some magnetohydrodynamic (MHD) activity triggered by the subsequent destabilization of $m=n=1$ internal modes. Igochine and ASDEX's coworkers ${ }^{7,8}$ were recently able to extract the dominant MHD activity during a sawtooth cycle. They observed that the whole cycle is dominated by an $m=n=1$ mode that survives the sawtooth crash, that is whose amplitude does not vanish as a result of the crash, which is consistent with an incomplete reconnection. During the sawtooth cycle, some $m=n=2$ mode is also present and grows from an almost negligible amplitude just after the crash up to one fourth of the $m=n=1$ amplitude ${ }^{7}$. An $m=n=3$ mode is also detected just before the sawtooth crash at a much lower amplitude. The crash phase was then interpreted as a consequence of the stochastization of the magnetic field lines.

One objective of the present article is to propose an explanation for the chronology of the onset of these $m=n$ modes. This is addressed in Section II through a reduced 
MHD approach. Section III then focuses on the question of the nonlinear development of the axisymmetric $m=n=0$ magnetic component or, equivalently, of the nonlinear evolution of the $q$-profile. Finally, the previous analytical results obtained in a simplified framework are connected to experimental measurements in Section IV. The role played by the local flattening of the $q$-profile around the $q=1$ surface is emphasized.

\section{DEVELOPMENT OF $m=n$ MODES INTO THE NONLINEAR REGIME}

\section{A. Principles of the analysis}

As it is well known, the toroidal geometry induces a coupling between all the $m$-numbers for a given $n$. Consequently, in the linear regime, the instability of the internal $m=n=1$ mode goes along, in particular, that of the $m=2, n=1$ secondary mode. In this Section, we shall however focus only on the development of the $m=n$ modes that are the dominant modes and, for the description of which, the cylindrical approach is sufficient.

Let us then study the development of MHD internal $m=n$ modes into the nonlinear regime starting from a linearly unstable $m=n=1$ mode. Defining by $A$ the amplitude of the $m=n=1$ mode, one can address this problem through an amplitude expansion. Looking back at the literature on this approach, it happens to have been first considered in fluid mechanics by Stuart in a seminal study of the nonlinear mechanics of instabilities in plane Poiseuille flow and Taylor-Couette flow ${ }^{9}$. More recently, Dahlburg applied similar techniques to determine the nonlinear evolution of some linearly unstable magnetohydrodynamic settings ${ }^{10}$. Independently, a comparable amplitude expansion approach was used later $^{11-13}$ to study the onset of the nonlinear regime of the internal resistive $m=n=1$ mode including the issue of the generation of higher harmonics of the disturbance that was left apart in Refs. 9,10. This question will be reconsidered more carefully now.

To address the issue of the development into the nonlinear regime of internal $m=n$ modes, one shall naturally proceeds through the following steps:

i) Only the $m=n=1$ mode is linearly unstable. Due to unavoidable nonideal effects, the plasma domain divides in two regions: an ideal region away from the $q=1$ surface in which nonideal effects are negligible and a thin current sheet region in the critical layer in the vicinity of the $q=1$ surface, in which nonideal effects deeply modify the local plasma dynamics and are responsible for the instability. The linear eigenfunction will result then from the matching between an outer ideal solution and an inner solution valid inside the critical layer.

ii) Then one injects this linear solution into MHD equations and look at the outcome on the various $m=n$ modes. Typically quadratic nonlinear terms may contribute to the $m=n=0$ and $m=n=2$ modes but do not affect the $m=n=1$ mode that will be only affected at the next order.

iii) There are two ways to consider step ii): one may assume that the critical layer is fixed or not. If the position of the critical layer is fixed, or assumed to be so, one can practically restrict to the critical layer equations and apply step ii) within the critical layer. This would practically correspond to the setting imposed in many slab numerical MHD simulations studying the behavior of e.g. tearing modes into the nonlinear regime. One may then refer to Stuart's approach ${ }^{9}$ for an analytic method to study the nonlinear evolution of the $m=n=1$ mode. Otherwise, one has to deal with a free layer problem and take into account the impact of the motion and possible modifications of the critical layer as time proceeds. This becomes an obviously more intricate problem which is a priori closer to the reality of the internal mode dynamics in tokamak plasmas. The later case will be considered in the following. Then nonlinear mode couplings will be shown to come into play in the first place due to the terms appearing as a result of the motion of the layer whereas the basic linear structure of the critical layer equations shall be conserved.

\section{B. The reduced MHD framework}

Practically, we consider cases relevant to hot tokamak plasmas operating close to the ideal MHD stability limits so that the $m=n=1$ internal kink mode is marginally stable against ideal MHD. Non-ideal effects, such as resistivity, may however turn it unstable. In order to explain the development of $m=n$ modes into the nonlinear regime, a prototypical relevant framework to consider is the reduced MHD system given by

$$
\begin{aligned}
& \frac{\partial U}{\partial t}=[\phi, U]+[J, \psi], \\
& \frac{\partial \psi}{\partial t}=[\phi, \psi]+\eta\left(J-J_{0}\right),
\end{aligned}
$$

where the standard definitions have been used. In the cylindrical geometry considered, the $(1,1)$ internal mode is ideally marginally stable.

In Eqs. (1)-(2), helical symmetry is assumed: The poloidal and toroidal angles, respectively $\theta$ and $\varphi$, only come in through the helical angle $\alpha=\varphi-\theta$. $\phi$ and $\psi$ are the plasma velocity and helical magnetic field potentials: the velocity is $\mathbf{v}=\hat{\varphi} \times \nabla_{\perp} \phi$ and the magnetic field $\mathbf{B}=B_{0 \varphi} \hat{\varphi}+\hat{\varphi} \times \nabla_{\perp}\left(\psi-r^{2} / 2\right) . U=\nabla_{\perp}^{2} \phi$ is the vorticity and $J=\nabla_{\perp}^{2} \psi$ the helical current density, with $\nabla_{\perp}^{2} \equiv r^{-1} \partial_{r} r \partial_{r}+r^{-\overline{2}} \partial_{\alpha}^{2}$. Poisson brackets are defined by $[\phi, U]=-\hat{\varphi} \cdot\left(\nabla_{\perp} \phi \times \nabla_{\perp} U\right)=r^{-1}\left(\partial_{r} \phi \partial_{\alpha} U-\partial_{r} U \partial_{\alpha} \phi\right)$. Eqs. (1)-(2) are dimensionless: Time has been normalized to the poloidal Alfvén time, the radial variable $r$ to the minor radius, and $\eta$ is the inverse of the magnetic Reynolds number $S$, and is given by the ratio of the poloidal Alvén time to the resistive one. In hightemperature fusion plasmas, $\eta$ is typically much smaller 
than one (of the order $10^{-8}$ ). Note that in this Section, modes will be labeled by their sole helical number $m$, which is equivalent to the usual notation in terms of poloidal and toroidal numbers $(m, n)$ with the restriction $|m|=|n|$.

Consider equilibria such that, for some internal radius $r_{s 0}<1, q\left(r_{s 0}\right)=1$, that is $\psi_{0}^{\prime}\left(r_{s 0}\right)=0$. Then, due to the Ohm's law (2), plasma volume divides in two region. Far from the $q=1$ surface (outer domain), plasma behaves ideally whereas, in the vicinity of $r_{s 0}$ (inner region), resistivity plays a crucial, destabilizing, role. Linear theory ${ }^{14}$ uses asymptotic matching analysis to provide $m=1$ eigenfunctions in the form $A(t) f_{L}(r) e^{i \alpha}$ valid in the whole domain. In the outer (ideal) domain, this solution is valid, that is nonlinear effects are negligible, as long as $A \ll 1^{12}$. Injecting the linear solutions $\psi_{1}(r, \alpha, t)=A(t) \psi_{L}(r) e^{i \alpha}$ and $\phi_{1}(r, \alpha, t)=A(t) \phi_{L}(r) e^{i \alpha}$ into (1)-(2) calls for an amplitude expansion. The procedure has been given in Refs. 11,12. The particularity of the linear radial eigenfunctions $\psi_{L}(r)$ and $\phi_{L}(r)$, that needs a careful consideration, is that they have strong gradients inside the critical layer. More precisely, their radial derivatives are of the order of the inverse of the critical layer width, that is $\mathcal{O}\left(\eta^{-1 / 3}\right)$.

\section{Linear solution}

\section{Linear solution in the outer domain}

In the linear regime, the helical magnetic field in the outer domain, namely out of the resistive critical layer, reads

$\psi^{\text {out }}(r, \alpha, \tau)=\left\{\begin{array}{c}\psi_{0}(r)+A(\tau) \psi_{L}(r) e^{i \alpha}+c . c . \text { for } r<r_{s 0} \\ \psi_{0}(r) \text { for } r_{s 0}<r \leq 1\end{array}\right.$

where the radial part $\psi_{L}(r)$ is a solution of the Newcomb equation

$$
\nabla_{\perp}^{2} f=f^{\prime \prime}+\frac{1}{r} f^{\prime}-\frac{f}{r^{2}}=\frac{d J_{0}}{d r}\left(\frac{d \psi_{0}}{d r}\right)^{-1} f
$$

for $r<r_{s 0}$ with the boundary condition $\psi_{L}^{\prime}\left(r_{s 0}^{-}\right)=1$. Considering the outer MHD equations, the outer solution (3) is valid as long as nonlinear effects may be neglected, namely as long as the $m=1$ mode amplitude $A$ is small compared to 1 . This outer linear solution provides then the matching boundary conditions for the following critical layer analysis as long as $A(\tau)$ is small (compared to one).

\section{Linear solution within the resistive critical layer}

The linear theory of the resistive mode was first derived by Coppi et al. in Ref. 14. A scaling analysis was given in Ref. 12 and the main results will be summarized below.
Let us define the stretched critical layer coordinate $x=\eta^{-1 / 3}\left(r-r_{s 0}\right)$ and the rescaled time $\tau=\eta^{1 / 3} t$ and denote by $w=\eta^{1 / 3}$ the (order of) the width of the critical layer. Noting that $J_{1} \sim \eta^{-2 / 3} \partial^{2} \psi_{1} / \partial x^{2}$ and $U_{1} \sim \eta^{-2 / 3} \partial^{2} \phi_{1} / \partial x^{2}$, one obtains at leading order the linear system of equations

$$
\begin{aligned}
\frac{\partial}{\partial \tau} \frac{\partial^{2} \phi_{1}}{\partial x^{2}}+\frac{\psi_{0}^{\prime \prime}\left(r_{s 0}\right)}{r_{s 0}} x \frac{\partial^{3} \psi_{1}}{\partial \alpha \partial x^{2}} & =0 \\
\frac{\partial \psi_{1}}{\partial \tau}+\frac{\psi_{0}^{\prime \prime}\left(r_{s 0}\right)}{r_{s 0}} x \frac{\partial \phi_{1}}{\partial \alpha} & =\frac{\partial^{2} \psi_{1}}{\partial x^{2}}
\end{aligned}
$$

where we used $\partial \psi_{0} / \partial x \sim \eta^{2 / 3} x \psi_{0}^{\prime \prime}\left(r_{s 0}\right)$. We define

$$
\kappa_{0} \equiv \frac{\psi_{0}^{\prime \prime}\left(r_{s 0}\right)}{r_{s 0}}
$$

Looking for solutions of the form $\phi_{1} \sim \phi_{1}(x) e^{i \alpha-i \omega \tau}$ and $\psi_{1} \sim \psi_{1}(x) e^{i \alpha-i \omega \tau}$, one unstable solution is found, the $m=1$ resistive mode, with growth rate

$$
-i \omega=\hat{\gamma}_{L}=\kappa_{0}^{2 / 3}=\left[\frac{\psi_{0}^{\prime \prime}\left(r_{s 0}\right)}{r_{s 0}}\right]^{2 / 3}=q^{\prime}\left(r_{s 0}\right)^{2 / 3} .
$$

The variable $x$ enters the equations through the rescaled variable $\kappa_{0}^{1 / 3} x / \sqrt{2}$. In real space, the perturbed potentials are

$$
\begin{aligned}
& \psi_{1}(x, \alpha, \tau)=A_{0} \exp \left(\hat{\gamma}_{L} \tau\right) g_{L}\left(\frac{\kappa_{0}^{1 / 3} x}{\sqrt{2}}\right) \cos \alpha \\
& \phi_{1}(x, \alpha, \tau)=-\frac{A_{0}}{\sqrt{2}} \exp \left(\hat{\gamma}_{L} \tau\right) g_{L}^{\prime}\left(\frac{\kappa_{0}^{1 / 3} x}{\sqrt{2}}\right) \sin \alpha
\end{aligned}
$$

where $g_{L}$ is the function

$$
g_{L}(s)=\frac{s}{2} \operatorname{erfc}(s)-\frac{1}{2 \sqrt{\pi}} \exp \left(-s^{2}\right) .
$$

This solution was chosen to satisfy the asymptotic matching conditions, giving $g_{L}^{\prime}(-\infty)=1$ and $g_{L}^{\prime}(+\infty)=0$.

These linear equations (5)-(6) are the dominant equations provided the nonlinear terms are effectively negligible. The validity of the linear system (5)-(6) breaks when the amplitude $A$ is no longer small compared to $\eta^{2 / 3}$, that is when the nonlinear terms, such as $\left[\phi_{1}, U_{1}\right]$ going like $\eta^{-1} A^{2}$, begin to dominate the linear ones, scaling as $\kappa_{0} \eta^{1 / 3} x \partial_{\alpha} J_{1} \sim A \eta^{-1 / 3}$. Alternatively, the end of validity of the linear regime for $A \gtrsim \eta^{2 / 3}$ coincides with the instantaneous location of the critical layer $r_{s}(\alpha, \tau)$, or with the width of the $m=1$ magnetic island, going out the initial critical layer centered on $r_{s 0}$.

\section{Onset of nonlinearities}

In writing down the critical layer equations, the instantaneous surface $r_{s}(\alpha, t)$, defined as the surface of vanishing transverse magnetic field by $\partial_{r} \psi\left[r_{s}(\alpha, t)\right]=0$, 
is important as the location where dynamical equations turn singular and non-ideal (resistive) effects come into play. Let us now estimate the location of the critical region at the end of validity of the linear stage. Writing $r_{s}(\alpha, \tau)=r_{s 0}+r_{s 1}(\alpha, \tau)$, we get

$$
d_{r} \psi_{0}\left(r_{s 0}+r_{s 1}(\alpha, \tau)\right)+\partial_{r} \psi_{1}\left(r_{s 0}+r_{s 1}(\alpha, \tau)\right)=0
$$

giving the first order correction $r_{s 1}(\alpha, \tau) \sim$ $A(\tau) / \eta^{1 / 3} \cos \alpha$. This relates to the first order shift of the core plasma inside the $q=1$ surface as a result of the kink instability.

Mode couplings do not affect the second order $m=1$ dynamics, so that one can keep the linear $m=1$ radial structure but introduce the corrections due to the motion of the $\partial_{r} \psi=0$ surface. Mode couplings only affect the $m=0$ and $m=2$ modes. The dynamical equations along the $m=0$ and $m=2$ components were derived in Ref. 12. Let us first focus on the evolution of the $m=2$ perturbation and define $y=\left(r-r_{s}(\alpha, \tau)\right) / w$ with $w=\eta^{1 / 3}$. At leading order in $w$, the dynamical equations projected on this component are

$$
\begin{aligned}
\frac{\partial}{\partial \tau} \frac{\partial^{2} \phi^{(2)}}{\partial y^{2}}+2 i \kappa^{(0)} y \frac{\partial^{2} \psi^{(2)}}{\partial y^{2}} & =w \frac{\partial r_{s 1}^{(1)}}{\partial \tau} \frac{\partial U_{1}^{(1)}}{\partial r} \\
\frac{\partial \psi^{(2)}}{\partial \tau}+2 i \kappa^{(0)} y \phi^{(2)}-\frac{\partial^{2} \psi^{(2)}}{\partial y^{2}} & =w^{-1} \frac{\partial r_{s 1}^{(1)}}{\partial \tau} \frac{\partial \psi_{1}^{(1)}}{\partial r}
\end{aligned}
$$

Due to the motion of the critical layer, the linear operators now involve the $m=0$ time-dependent average

$$
\kappa^{(0)}(\tau) \equiv \frac{1}{2 \pi} \int_{0}^{2 \pi} \frac{\psi^{\prime \prime}\left[r_{s}(\alpha, \tau)\right]}{r_{s}(\alpha, \tau)} d \alpha
$$

instead of $\kappa_{0}$. The system (13)-(14) consists in a linear kernel forced by convective terms. As the $m \neq 1$ components of the perturbations have amplitudes that are assumed to be initially almost vanishing, it will be valid to neglect their importance in front of the $m=1$ mode. Following Eqs. (13)-(14), the qualitative time behavior of the forcing term due to the motion of the layer is $A d A / d \tau$. Therefore the approximation for the time behavior of the $m=2$ mode will be qualitatively given by $A_{0}^{2} \hat{\gamma}_{L} \tau \exp \left(2 \hat{\gamma}_{L} \tau\right)$. Consequently, the $m=1$ mode is dominant in the early nonlinear regime over the nonlinearly triggered $m=2$ mode as long as $\tau \ll \tau_{s}$ with

$$
\hat{\gamma}_{L} \tau_{s}=-\ln A_{0}-\ln \left(\hat{\gamma}_{L} \tau_{s}\right) .
$$

This will typically be the case during many e-folding times provided that the initial perturbation amplitude $A_{0}$ is low enough. Let us note that conversely, in the case where the initial $m=1$ mode amplitude is not sufficiently small, the linear regime may not be observed at all.

Once the $m=2$, and possibly also the $m=0$, modes are nonlinearly destabilized, this gives way to the nonlinear cascade of mode couplings: the $m=3$ mode is nonlinearly triggered through mode couplings involving the $m=2$ and $m=1$ modes as well as cubic $m=1$ terms, and so on. Let us now investigate the evolution of the $m=0$ mode as a result of nonlinearities.

\section{THE $\mathrm{M}=\mathrm{N}=0$ MODE: EVOLUTION OF THE $q$-PROFILE}

\section{A. Critical layer equations for the $m=n=0$ perturbation}

Following continuously the linear regime through the introduction of the instantaneous critical surface $r_{s}(\alpha, \tau)$, we can now write down the dynamical equations in the corresponding moving critical layer projected on the $m=$ 0 component as

$$
\begin{aligned}
\frac{\partial}{\partial \tau} \frac{\partial^{2} \delta \phi^{(0)}}{\partial y^{2}} & =w \frac{\partial r_{s 1}^{(-1)}}{\partial \tau} \frac{\partial U_{1}^{(1)}}{\partial r}+c . c . \\
\frac{\partial \delta \psi^{(0)}}{\partial \tau}-\frac{\partial^{2} \delta \psi^{(0)}}{\partial y^{2}} & =w^{-1}\left(\frac{\partial r_{s 1}^{(-1)}}{\partial \tau} \frac{\partial \psi_{1}^{(1)}}{\partial r}+\frac{i}{r} \frac{\partial \phi_{1}^{(-1)}}{\partial r} \psi_{1}^{(1)}\right)+(c 1 \bar{c} .)
\end{aligned}
$$

Here the $m=0$ fields, $\delta \phi^{(0)}$ and $\delta \psi^{(0)}$, have to be considered as second order perturbations to their respective equilibrium fields. As we are specifically interested into the $q$-profile evolution, we shall just consider Eq. (17). One should be careful that the first order terms $\psi_{1}$ and $U_{1}$ correspond to the linear solution previously derived and do not have large derivatives outside the linear critical layer centered on $r_{s 0}$.

The right-hand side of Eq. (17) involves the sum of two quadratic convective terms, the first one of which being due to the motion of the critical layer. It is easy to realize that this one dominates the other one when $w^{-1} d A / d \tau \gg$ $A$ which is clearly the case in the early nonlinear regime for which $A \simeq A_{0} \exp \left(\hat{\gamma}_{L} \tau\right)$. This ordering legitimates the moving layer analysis. Eq. (17) then reads

$$
\frac{\partial \delta \psi^{(0)}}{\partial \tau}-\frac{\partial^{2} \delta \psi^{(0)}}{\partial y^{2}}=w^{-2} \frac{A(\tau)}{\sqrt{2}} g_{L}^{\prime}\left(\frac{\kappa_{0}^{1 / 3} y}{\sqrt{2}}\right) \kappa_{0}^{1 / 3} \frac{1}{2 \pi} \int_{0}^{2 \pi} \frac{\partial r_{s}(\alpha, \tau)}{\partial \tau}
$$

One recognizes a diffusion equation with sources for $\delta \psi^{(0)}$. Putting $s=0$ would yield a simple diffusion equation with vanishing boundary conditions yielding an identically vanishing $\delta \psi^{(0)}$. The $\alpha$-dependence of the critical radius when $A>\eta^{2 / 3}$, due to the $m=1$ perturbation breaking the radial symmetry, is the main ingredient that triggers nonlinearly the onset of the $m=0$ perturbation. Therefore the moving critical layer model is consistent enough to avoid any invocation of some prescribed background turbulence that would feed $m=0$ and $m=2$ components. The only necessary assumption is the existence at time zero of some infinitesimal amplitude of the $m=1$ resistive internal mode. 

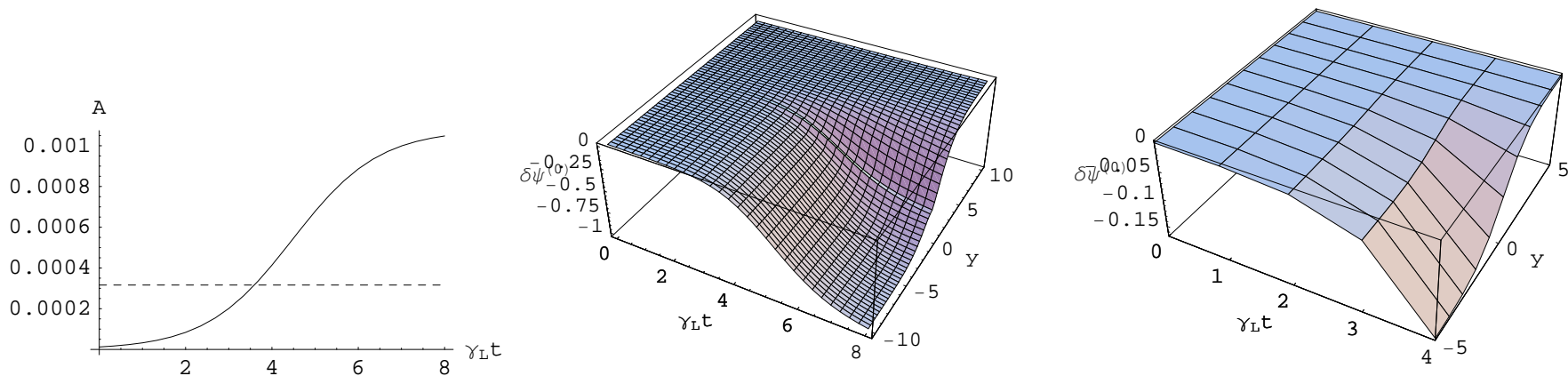

FIG. 1. (left panel) Time evolution of the amplitude $A$ of the $m=1$ resistive mode derived from the nonlinear model ${ }^{11-13}$; the dashed line indicates the threshold for the onset of cubic nonlinearities $\left(A \sim \eta^{1 / 2}\right.$ ), and (central panel) corresponding time evolution of the perturbation of the axisymmetric toroidal magnetic flux obtained from the solution (19) of the critical layer diffusion equation with (right panel) a zoom on the early evolution.

\section{B. Time evolution of the $m=n=0$ perturbation within the resistive internal layer}

Eq. (18) is solved for $\delta \psi^{(0)}(y, \tau=0)=0$, with $-\infty<$ $y<\infty$, and homogeneous boundary conditions, as

$$
\delta \psi^{(0)}(y, \tau)=\int_{0}^{\tau} \int_{-\infty}^{\infty} \frac{s(z, \iota)}{\sqrt{4 \pi(\tau-\iota)}} \exp \left[-\frac{(y-z)^{2}}{4(\tau-\iota)}\right] d \iota d z .
$$

In Eq. (19), the sources term scales as $A^{2} w^{-2}$, so that the $m=0$ perturbation of the helical magnetic flux within the moving critical layer becomes of order one for $A \sim$ $w=\eta^{1 / 3}$. This corresponds to the threshold predicted for nonlinear saturation in Refs. 11,12.

Before turning to the numerical evaluation of the $\delta \psi^{(0)}$ evolution, it is important to note that $\delta \psi^{(0)}$ is not the $m=0$ perturbation of the magnetic helical flux in the cylinder variables. It is so relative to the instantaneous critical layer and with respect to the reduced coordinate $y$ that depends on $\alpha$. This would have to be taken into account for the final evaluation.

The right plot of Figure 1 presents the numerical evaluation of the perturbation of $\psi^{(0)}$ for some special case. It has been estimated by plugging for $\psi_{1}$ the linear eigenfunction inside the critical layer, so that the result remains correct as long as $A$ is not too large but overestimates the true result in the late times. Initial conditions are the same as in Aydemir's paper ${ }^{15}$. The chosen qprofile is $q_{R}(r)=q_{0} \sqrt{1+\left[\left(\frac{q_{a}}{q_{0}}\right)^{2}-1\right] r^{4}}$, with $q_{0}=0.9$ and $q_{a}=3$. Correspondingly, the radius of the location of the $q=1$ surface is initially $r_{s 0}=0.39$. The magnetic Reynolds number is $S=\eta^{-1}=10^{7}$. The initial $m=1$ mode amplitude is $1.2 \times 10^{-5}$.

The left side of Figure 1 displays for reference the solution for the $m=1$ amplitude of the generalized critical layer dynamical equations projected on the $m=1 \mathrm{com}-$ ponent, namely the solution of

$$
\frac{1}{\kappa^{(0)}(\tau)} \frac{1}{A} \frac{d A}{d \tau}=\frac{\gamma_{L}}{\kappa_{0}}=\kappa_{0}^{-1 / 3}
$$

with the instantaneous critical layer average

$$
\kappa^{(0)}(\tau)=\frac{1}{2 \pi} \int_{0}^{2 \pi} \frac{\psi_{0}^{\prime \prime}\left(r_{s}(\alpha, \tau)\right)}{r_{s}(\alpha, \tau)} d \alpha .
$$

From the computations of the $m=0$ helical magnetic

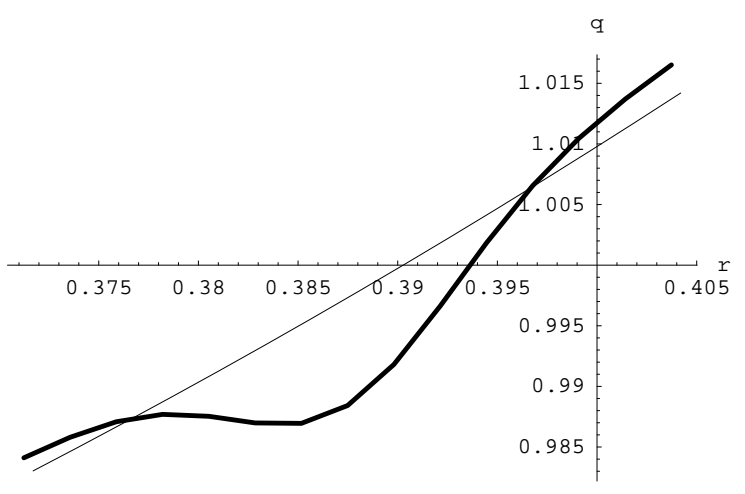

FIG. 2. Prediction for the early $q$-profile modification within the resistive critical layer obtained from Eq. (19) for the same parameters as in Fig. 1. The plain curve represents the initial reference equilibrium $q$-profile and the bold curve the $q$-profile computed at time $\gamma_{L} t=1$.

perturbation in the inner resistive layer displayed in Figure 1 , it is then possible to derive the early nonlinear modification of the $q$-profile. This is indicated in Figure 2 . We have here used the following definition for the instantaneous $q$-profile given by $d \psi_{0} / d r=r(1 / q(r, \tau)-1)$, with $\psi_{0}=\psi_{0 R}+\delta \psi^{(0)}(r, \tau)$ and $d \psi_{0 R} / d r \equiv r\left(1 / q_{R}(r)-\right.$ 
1). The $q$-profile then just translates the fate of the axisymmetric magnetic component. From this follows that the plateau associated to the intrinsically nonaxisymmetric $m=n=1$ magnetic island in Figure 2 is associated to a value of $q$ (slightly) smaller than one and not equal to one.

\section{Outer $q$-profile evolution}

Outside the critical resistive layer, in the approximately ideal domain, the Ohm's law projected on the $m=0$ mode amounts to the diffusion equation $\partial \delta \psi^{(0)} / \partial t=\eta \delta J^{(0)}$, namely

$$
\frac{\partial \delta \psi^{(0)}}{\partial \tau}=\frac{w^{2}}{r}\left[\frac{\partial}{\partial r}\left(r \frac{\partial \delta \psi^{(0)}}{\partial r}\right)\right] .
$$

This equation has to be solved in both radial domains $I_{-} \equiv\left[0 ; r_{s}^{-}(\tau)\right]$ and $I_{+} \equiv\left[r_{s}^{+}(\tau) ; 1\right]$. In $I_{-}$, the solution should satisfy $\lim _{r \rightarrow 0}\left|\delta \psi^{(0)}(r, \tau)\right|<\infty$ and should match the critical layer solution through $\delta \psi^{(0)}\left(r_{s}^{-}(\tau), \tau\right)=\lim _{y \rightarrow-\infty} \delta \psi_{i n}^{(0)}(\tau) \equiv g^{-}(\tau)$. In $I_{+}$, the boundary conditions are $\delta \psi^{(0)}\left(r_{s}^{+}(\tau), \tau\right)=$ $\lim _{y \rightarrow+\infty} \delta \psi_{i n}^{(0)}(\tau) \equiv g^{+}(\tau)$ and $\delta \psi^{(0)}(r=1, \tau)=0$. In both cases, the initial condition is $\delta \psi^{(0)}(r, 0)=0$. Therefore, the present problem to solve is that of a diffusion equation in cylindrical coordinates with time dependent boundary conditions in which one of the boundaries is moving. The latter requirement makes the problem clearly non-standard. In order to get a physical insight of the solution, one can however neglect in the first place the motion of the boundaries and retain solely in the inner domain $\delta \psi^{(0)}\left(r_{s 0}^{-}, \tau\right)=g^{-}(\tau)$.

The features of the solution of Eq. (22) are easy to derive. Compared to the timescale of the critical layer dynamics, the timescale of the diffusion of $\delta \psi^{(0)}$ is typically much larger, so that, provided $\eta$ is small enough, $\delta \psi^{(0)}$ should remain vanishingly small except in the vicinity of the inner border of the critical layer boundary where it decreases to match the inner solution.

Let us now solve Eq. (22) and denote by $\delta \hat{\psi}^{(0)}(r, s)$ the Laplace transform of the function $\delta \psi^{(0)}(r, t)$. Proceeding to the Laplace transform of the diffusion equation Eq. (22) and boundary conditions yields

$$
\frac{w^{2}}{r}\left[\frac{\partial}{\partial r}\left(r \frac{\partial \delta \hat{\psi}^{(0)}}{\partial r}\right)\right]-s \delta \hat{\psi}^{(0)}(r, s)=0,
$$

for $0 \leq r<r_{s 0}^{-}$, with

$$
\lim _{r \rightarrow 0}\left|\delta \hat{\psi}^{(0)}(r, s)\right|<\infty,
$$

and

$$
\delta \hat{\psi}^{(0)}\left(r_{s 0}^{-}, s\right)=\hat{g}^{-}(s)
$$

It is obviously very important that the physical condition (24) be satisfied, a fact that may not be fulfilled by some (bad) parabolic partial differential equation solvers.

The solution to this boundary-value problem in cylindrical geometry is (see e.g. Ref. 16)

$$
\delta \hat{\psi}^{(0)}(r, s)=\hat{g}^{-}(s) \frac{I_{0}\left(\frac{r \sqrt{s}}{w}\right)}{I_{0}\left(\frac{r_{s 0}^{-} \sqrt{s}}{w}\right)} .
$$

Then it remains to proceed to the inverse Laplace transform of $\delta \hat{\psi}^{(0)}(r, s)$ given in Eq. (26) to obtain $\delta \psi^{(0)}(r, \tau)$ and the associated $q$-profile.

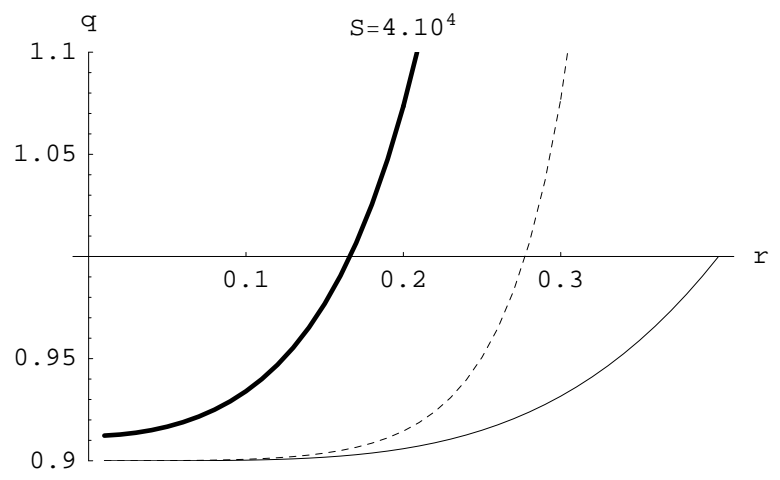

FIG. 3. Predicted $q$-profile time evolution in the core plasma given by the numerical solution of the diffusion equation (22) for a fixed maximal boundary and a moderate Lundquist number, namely $S \equiv \eta^{-1}=4.10^{4}$. The plain curve represents the initial equilibrium $q$-profile, the dashed curve the $q$-profile computed at time $\gamma_{L} t=7$, and the bold curve the $q$-profile computed at time $\gamma_{L} t=14$. The parts of the $q$-profile above one may be irrelevant since, correspondingly to the growth of the magnetic island width, the boundary $r_{s}^{-}(\tau)$ diminishes and shifts towards the axis.

Figure 3 shows the predicted early time evolution of the $q$-profile corresponding to the numerical integration of the diffusion equation (22) for a moderate value of the Lundquist number, namely $S \sim 4 \times 10^{4}$, similar to the one used in Ref. 17. Inverse Laplace transforms of Eq. (26) were numerically computed using a Gaver-WynnRho algorithm ${ }^{18}$. As expected from Section III B, the overestimation of the boundary value $g^{-}(\tau)$ in the late second order regime may explain the overestimation of the $q$-profile close to the critical layer border. Moreover, one should not forget that, in order to simplify the problem, we have solved here the diffusion equation in the inner core plasma by assuming a fixed boundary at $r=r_{s 0}$ which is certainly not valid as time increases due to the growth of the magnetic island. The effect of this growth of the magnetic island will be to lower the effective boundary $r_{s}^{-}(\tau)$ as time increases so that, at time $\tau$, one may discard the results in the computational zone $\left[r_{s}^{-}(\tau), r_{s 0}\right]$ in which the obtained $q$ values are typically larger than one. 
Having said that, one can now interpret this computation of the $q$-profile evolution. This is important because this gives in particular the time evolution of the central value, $q_{0}$, of the $q$-profile, the behavior of which being a well-known indicator of the complete or incomplete character of the magnetic reconnection. Only for moderate $S$ values possibly relevant to describe sawteeth in small tokamaks does one observe the slow progressive growth of $q_{0}$ as in Figure 3. It is interesting to mention that in Ref. 17, Watanabe et al. observed the progressive, and eventually complete, flattening of the core current profile in the course of the nonlinear development of the resistive kink mode for the resistivity value considered in this Figure. However, for the realistically large values of $S$ expected in fusion-relevant tokamak plasmas, one predicts on the basis of the solution (26) that $q_{0}$ should remain practically unchanged and equal to its initial equilibrium value in the early nonlinear regime. This favors an incomplete reconnection.

\section{Summary and comments on the results}

In the present case, only the $m=n=1$ mode has been considered to be linearly unstable. In the linear regime, its amplitude is of the form $A(t)=A_{0} e^{\gamma_{L} t}$. This is obviously valid as long as $A$ is small enough. Injecting this linear solution into the full (nonlinear) resistive MHD equations, it can be seen that the first dominant nonlinear terms obtained are quadratic in $A$ and affect the modes $m=1 \pm 1$, namely the modes $m=0$ and $m=2$. Later the cubic terms will affect the modes $0 \pm 1$ and $2 \pm 1$, namely the modes $m=1$ and $m=3$. At small enough amplitudes (here for $A \ll 1$ ), the outer plasma can be treated linearly. This means that nonlinearities will at first only affect the critical layer in which resistive effects are important, driving the dynamics of the whole plasma by providing the boundary conditions that must be satisfied by the outer fields. This has been exemplified in Section III C through the computation of the time evolution of the $q$-profile in the plasma center.

We expect the scenario of the successive nonlinear switching-on of modes as $A$ increases to be very general. Here the mode cascade proceeds as $A$ crosses thresholds in the resistivity $\eta$. This can be viewed as the entrance door into the later turbulent regime where a large number of modes have been activated. The limitation of the present perturbative approach lies in the fact that the nonlinear width of the critical layer is assumed to be equal to $w$, namely the linear one. This continuity argument is fine to describe the early nonlinear regime. Later, the derived critical layer equations should remain valid only in some $\alpha$ domain close to the x-point of the $m=1$ island. Practically, as long as the $m=1$ island width is not much larger than $w$, the assumption is correct. This is the case in the computation of the $q$-profile nonlinear modification within the resistive layer presented in Fig. 2 .

\section{IMPLICATIONS}

\section{A. Connection to the experimental measurements during a sawtooth cycle}

The mode amplitude analysis predicts that, at later times, when the magnitude of the $m=n=1$ mode amplitude becomes larger than $\eta^{1 / 3}$, cubic nonlinearities will become no longer negligible. In the cylindrical approach considered here, the cubic nonlinearities will destabilize the $m=3$ mode and affect the dynamics of the $m=1$ component but will affect the evolution equations for the $m=0$ and $m=2$ modes only through boundary conditions.

Let us observe that this is fully consistent with the picture of the sawtooth cycles reported e.g. in Refs. 7,8. Their experimental diagnostics reveal that a cycle begins with a non-negligible $(m, n)=(1,1)$ mode. As the amplitude of this mode grows, the $(m, n)=(2,2)$ mode starts to appear. Just before the crash, the $(m, n)=(3,3)$ mode is also observed although at a very small amplitude. Just after the very abrupt crash, the $(m, n)=(1,1)$ mode is detected, which suggests an incomplete reconnection, and the cycle repeats. This phenomenology is consistent with the previous mode amplitude analysis of the onset of the nonlinear regime that does predict the same chronology in the mode appearance.

Igochine $e t a l$. only report the existence of $m=n$ internal modes ${ }^{19}$, as would be predicted by a purely cylindrical approach. However, in toroidal geometry, the linear regime is more complex. In particular, the $m=2, n=1$ and $m=0, n=1$ sideband modes are also linearly unstable but their amplitude may remain small in front of the $m=n=1$ one. One would then expect the generic realistic toroidal picture of the onset into the nonlinear regime to be more complex with the emergence of modes nonlinearly triggered due to mode couplings between the linearly unstable modes. Yet, the contribution from $m=n$ modes should remain dominant. Nevertheless, in order for the magnetic field lines to be stochastic, let us observe that it is necessary that modes having $m \neq n$, i.e. modes with different helicities, be present as exposed in the Appendix.

\section{B. Impact of the $q$-profile evolution on sawteeth}

The previous analysis (see Sec. IIIB) gives indication that the nonlinear development of the $m=1$ mode should be accompanied by a self-consistent perturbation of the $q$-profile inside the $q=1$ surface, so that the $q$ profile is no longer a strictly monotonous function but locally flattens or becomes reversed-shear. Such an indication is consistent with experimental observations. In particular, recent Alcator C-Mod experiments focusing on Alfvén eigenmodes in the current flattop phase of the discharge have discovered reversed shear Alfvén eigenmodes (RSAEs) near the $q=1$ surface between sawtooth 
crashes $^{20}$. RSAEs are known to exist near the minimum of the q profile in a reversed shear equilibrium and are driven unstable by the spatial gradient of the fast ion density from neutral beams, fusion born alpha particles, or in the case of Alcator C-Mod, an ICRH driven minority ion species.

It happens that the impact of the $q$-profile time variation has been neglected in most theoretical models of sawteeth. This may however be an important ingredient to explain the onset of the sawtooth crash, as will be briefly discussed below.

As it is well-known, because the magnetic field is globally divergence-free, its field lines can be identified with the phase-space trajectories produced by a magnetic field line Hamiltonian ${ }^{21}$, the derivation of which is sketched in the Appendix. The stochastic theory of the sawtooth crash proposed by Lichtenberg et al. ${ }^{22,23}$ relies on this formulation. As time proceeds, the magnetic islands associated to some modes with different helicities overlap and the magnetic phase space dynamics turns chaotic. In this picture, the sawtooth crash phase is associated to the sudden emergence of large scale chaos in the magnetic structure. In Refs. 24,25, it was shown that the local vanishing of the magnetic shear, namely due to a local flattening or reversed-shear $q$-profile, may have deleterious impact on the magnetic confinement if it takes place for a $q$ value in the vicinity of a small rational. Indeed this creates a double separatrix configuration ${ }^{24}$ that possesses a lower resilience to chaos than the ordinary single separatrix case: the emergence of chaos is facilitated by the merging of the two stochastic layers associated to the separatrices. We shall conclude this article by briefly illustrating this point.

Figure 4 shows two Poincaré plots of the magnetic field lines in a "pre-crash", low chaotic, situation for the same magnetic perturbations but two different $q$-profiles. In the left plot, the $q$-profile is a strictly monotonously increasing function with $q_{0}=0.8$ and $q(\psi=0.25)=1$. In the right plot, the $q$-profile is identical except that it is taken to be flat in a $\psi$ region of width 0.1 around $\psi=0.25$ for which it is taken equal to one. The resulting $q$-profile is made continuous and derivable. For simplicity, the radial dependence of the modes were chosen to correspond to the ideal MHD eigenmodes except for the nonlinearly triggered $(2,2)$ mode for which a phenomenological form was retained as in Refs. 4,5. The comparison between the left and right Poincaré plots of Figure 4 shows the impact of the local flattening of the $q$-profile in the $q=1$ region. In the case of the flattening at right, a slightly stochastic double separatrix configuration is discernable: a small crescent shaped island is visible in the outer vicinity of the traditional $m=1$ magnetic island which is absent in the left case of the strictly monotonously growing $q$ profile. This is the seed for the facilitation of chaos at larger mode amplitudes in the right case. This will be studied more closely in a forthcoming paper.

\section{ACKNOWLEDGMENTS}

MCF thanks P. Buratti and J. Graves for useful communications. Financial support from the ECOSMINCyT Research Grant No. A09E02 is gratefully acknowledged. WE acknowledges funding from Ecole Polytechnique through a Gaspard Monge Scholarship.

This work was carried out within the framework the European Fusion Development Agreement and the French Research Federation for Fusion Studies. It is supported by the European Communities under the contract of Association between Euratom and CEA. The views and opinions expressed herein do not necessarily reflect those of the European Commission.

${ }^{1}$ S. Von Goeler, W. Stodiek and N. Sauthoff, Phys. Rev. Lett. 33, 1201 (1974)

${ }^{2}$ T. P. Goodman, F. Felici, O. Sauter, J. P. Graves, and the TCV team, Phys. Rev. Lett. 106, 245002 (2011).

${ }^{3}$ T. C. Hender, J. C. Wesley, J. Bialek, A. Bondeson, A. H. Boozer, R. J. Buttery, A. Garofalo, T. P. Goodman, R. S. Granetz, Y. Gribov, O. Gruber, M. Gryaznevich, G. Giruzzi, S. Gunter, N. Hayashi, P. Helander, C. C. Hegna, D. F. Howell, D. A. Humphreys, G. T. A Huysmans, A. W. Hyatt, A. Iasyama, S. C. Jardin, Y. Kawano, A. Kellman, C. Kessel, H. R. Koslowski, R. J. La Haye, E. Lazzaro, Y. Q. Liu, V. Lukash, J. Manickam, S. Medvedev, V. Mertens, S. V. Mirnov, Y. Nakamura, G. Navratil, M. Okabayashi, T. Ozeki, R. Paccagnella, G. Pautsso, F. Porcelli, V. D. Pustovitov, V. Riccardo, M. Sato, O. Sauter, M. J. Schaffer, M. Shimada, P. Sonato, E. J. Strait, M. Sugihara, M. Takechi, A. D. Turnbull, E. Westerhof, D. G. Whyte, R. Yoshino, H. Zohm, and the ITPA MHD, Disruption and Magnetic Control Topical Group, Nucl. Fusion 47, S128 (2007).

${ }^{4}$ R Farengo, H. E. Ferrari, M.-C. Firpo, P. L. García-Martínez, and A. F. Lifschitz, Plasma Phys. Control. Fusion 54, 025007 (2012).

${ }^{5}$ R. Farengo, H. E Ferrari, P. García-Martínez, M.-C. Firpo, W. Ettoumi and A. F. Lifschitz, Nucl. Fusion 53, 043012 (2013).

${ }^{6} \mathrm{P}$. Buratti, private communication (2010).

${ }^{7}$ V. Igochine, O. Dumbrajs, H. Zohm, and ASDEX Upgrade Team, Nucl. Fusion 48, 062001 (2008).

${ }^{8}$ V. Igochine, J. Boom, I. Classen, O. Dumbrajs, S. Günter, K. Lackner, G. Pereverzev, H. Zohm, and ASDEX Upgrade Team, Phys. Plasmas 17, 122506 (2010).

${ }^{9}$ J. T. Stuart, J. Fluid Mech. 4, 1 (1958).

${ }^{10}$ R. B. Dahlburg, Phys. Plasmas 5 (1), 133-139 (1998).

${ }^{11}$ M.-C. Firpo and B. Coppi, Phys. Rev. Lett. 90, 095003 (2003).

12 M.-C. Firpo, Phys. Plasmas 11, 970 (2004).

${ }^{13}$ M.-C. Firpo, Phys. Lett. A 342, 263 (2005).

${ }^{14}$ B. Coppi, R. Galvão, M. N. Rosenbluth and P. H. Rutherford, Sov. J. Plasma Phys. 2, 3276 (1976).

${ }^{15}$ A.Y. Aydemir, Phys. Rev. Lett. 78, 4407 (1997).

${ }^{16}$ D. G. Duffy, Transform methods for solving partial differential equations, Chapman \& Hall/CRC eds (2004).

${ }^{17}$ K. Watanabe, T. Sato, and Y. Nakayama, Nucl. Fusion 35, 251 (1995).

${ }^{18}$ P. P. Valkó and J. Abate, Comput. Math. Appl. 48, 629-636 (2004).

${ }^{19}$ Note, however, that the mapping technique used in Refs. 7,8 contains the corresponding toroidal coupling.

${ }^{20}$ E. M. Edlund, M. Porkolab, G. J. Kramer, L. Lin, Y. Lin, and S. J. Wukitch, Phys. Plasmas 16, 056106 (2009).

${ }^{21}$ P.J. Morrison, Phys. Plasmas 7, 2279 (2000).

${ }^{22}$ A. J. Lichtenberg, Nucl. Fusion 241277 (1984).

${ }^{23}$ A.J. Lichtenberg, K. Itoh, S.-I. Itoh and A. Fukuyama, Nucl. Fusion 32495 (1992). 

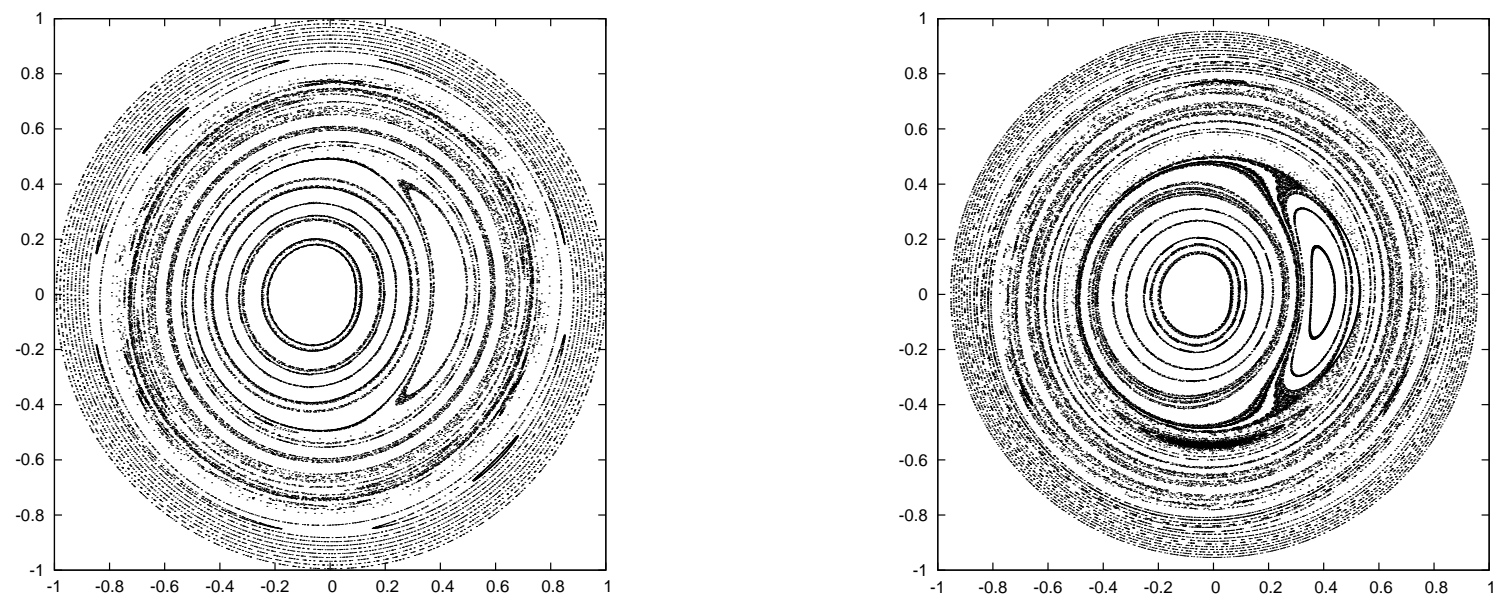

FIG. 4. Poincaré plots obtained for a moderate amplitude, $A$, of the $(1,1)$ mode and with the retained following mode amplitudes $A_{(2,1)}=0.2 A, A_{(3,2)}=0.05 A, A_{(4,3)}=0.02 A$ and $A_{(2,2)}=0.3 A$ for two different $q$-profiles: (left) monotonously increasing $q$-profile; (right) same $q$-profile but flattened around the $q=1$ surface as depicted in the text.

${ }^{24}$ M.-C. Firpo and D. Constantinescu, Phys. Plasmas 18, 032506 (2011).

${ }^{25}$ D. Constantinescu and M.-C. Firpo, Nucl. Fusion 52054006 (2012).

${ }^{26}$ A. H. Boozer, Phys. Fluids 26, 1288-1291 (1983).

\section{Appendix: Hamiltonian representation of magnetic field lines}

An arbitrary magnetic field in a toroidal device can be written in the canonical representation

$$
\mathbf{B}=\nabla \psi_{t} \times \nabla \theta+\nabla \varphi \times \nabla \Psi_{p}
$$

in which $\Psi_{p}\left(\psi_{t}, \theta, \varphi\right)$ is the poloidal flux function ${ }^{26}$. The magnetic field lines are defined by

$$
\begin{aligned}
\frac{d \psi_{t}}{d \varphi} & =\frac{\mathbf{B} \cdot \nabla \psi_{t}}{\mathbf{B} \cdot \nabla \varphi}=-\frac{\partial \Psi_{p}}{\partial \theta}, \\
\frac{d \theta}{d \varphi} & =\frac{\mathbf{B} \cdot \nabla \theta}{\mathbf{B} \cdot \nabla \varphi}=\frac{\partial \Psi_{p}}{\partial \psi_{t}} .
\end{aligned}
$$

In this representation, the poloidal flux plays the role of a generically one-and-a-half Hamiltonian system and the toroidal angle $\varphi$ plays the role of time. Due to the double periodicity in $\theta$ and $\varphi$, any function $\Psi_{p}$ can be written as

$\Psi_{p}\left(\psi_{t}, \theta, \varphi\right)=\Psi_{p_{00}}\left(\psi_{t}\right)+\sum_{m, n} \Psi_{p_{m n}}\left(\psi_{t}\right) \cos \left(m \theta-n \varphi+\chi_{m n}\right)$

where the equilibrium axisymmetric poloidal flux component $\Psi_{p_{00}}\left(\psi_{t}\right)$ relates to the safety profile $q\left(\psi_{t}\right)$ or to its inverse, the winding profile $w\left(\psi_{t}\right)$, through

$$
\frac{d \psi_{p_{00}}\left(\psi_{t}\right)}{d \psi_{t}}=\frac{1}{q\left(\psi_{t}\right)}=w\left(\psi_{t}\right)
$$

In order to connect to the previous helical description, let us now introduce the helical angle $\alpha=\varphi-\theta$ and proceed to a canonical change of variables with the generating function

$$
F_{2}\left(\psi^{\prime}, \theta, \varphi\right)=(\theta-\varphi) \psi^{\prime}
$$

This yields $\partial F_{2} / \partial \theta=\psi_{t}=\psi^{\prime}$ and $\partial F_{2} / \partial \psi=-\alpha=\theta-\varphi$. The new Hamiltonian reads

$$
H\left(\psi_{t}, \alpha, \varphi\right)=\Psi_{p_{00}}\left(\psi_{t}\right)-\psi_{t}+\sum_{m=n} \Psi_{p_{m n}}\left(\psi_{t}\right) \cos \left(m \alpha-\chi_{m n}\right)+\sum_{m \neq}
$$

In Eq. (A.3), we made use of the fact that the new action coordinate, $\psi^{\prime}$, is equal to $\psi_{t}$. One recovers the equilibrium component of the helical magnetic flux $\psi_{0}$ through

$$
\psi_{0}\left(\psi_{t}\right) \equiv \Psi_{p_{00}}\left(\psi_{t}\right)-\psi_{t}=\int^{\psi_{t}}\left(\frac{1}{q(s)}-1\right) d s
$$

so that, coming back to the radial coordinate through the cylindrical relation $\psi_{t}=r^{2} / 2$, one obtains the relation used in Sec. II B

$$
\frac{d \psi_{0}}{d r}=r\left(\frac{1}{q(r)}-1\right)
$$

From Eq. (A.3), one directly checks that the Hamiltonian of the magnetic field lines would be integrable in the absence of modes with different helicities. In this way, one may say that toroidal effects, together with non-ideal effects, are responsible for the generic non-integrability of magnetic field lines in tokamak plasmas. This representation is valid at any given time. 